\title{
On Perturbation Theory for Regularized Determinants of Differential Operators
}

\author{
R. E. Gamboa-Saravi`, M. A. Muschietti`, and J. E. Solomin` \\ Universidad Nacional de La Plata, 1900 La Plata, Argentina
}

\begin{abstract}
A perturbation theory for determinants of differential operators regularized through the $\zeta$-function technique is presented. The application of this approach to the study of chiral changes in the fermionic path-integral variables is discussed.
\end{abstract}

\section{Introduction}

In the Feynman path-integral approach to quantum theory one is naturally led to the computation of determinants of differential operators. These determinants clearly diverge because the eigenvalues $\lambda_{j}$ increase without bound. Therefore, it is necessary to adopt some regularization procedure. One technique which has proved to be very useful is the $\zeta$-function regularization [1]. When $A$ is an elliptic invertible operator of order $m>0$, defined on some compact manifold $M$ without boundary of dimension $n$, one forms a generalized $\zeta$-function from the eigenvalues $\lambda_{j}$ of $A$ :

$$
\zeta(s, A)=\sum_{j} \lambda_{j}^{-s}
$$

This series converges only for $\operatorname{Re} s>n / m$, but $\zeta(s, A)$ can be analytically extended to a meromorphic function of $s$ in the whole complex plane [2]. In particular it is regular at $s=0$. The derivative of the $\zeta$-function at $s=0$ is formally equal to $-\sum_{j} \log \lambda_{j}$. One can therefore define the regularized determinant of $A$, $\operatorname{Det}(A)$, to be $\left.\exp (-d \zeta / d s)\right|_{s=0}$.

The purpose of this paper is to study the behavior of $\operatorname{Det}(A)$ when the operator $A$ is perturbed by another operator of smaller order. To be more precise, we are going to prove in Sect. 2 that

$$
\operatorname{Det}\left(A+\sum_{j=1}^{p} \varepsilon^{j} A_{j}\right)=\operatorname{Det}(A) \exp \left[\varepsilon c_{1}+\varepsilon^{2} c_{2}+\ldots+\varepsilon^{r} c_{r}+O\left(\varepsilon^{r+1}\right)\right]
$$

* Member of Comisión de Investigaciones Científicas de la Provincia de Buenos Aires, Argentina 
when $\operatorname{ord}\left(A_{j}\right)<\operatorname{ord}(A) j=1,2, \ldots, p$. The coefficients $c_{j}$ can, in principle, be computed in terms of the operators $A$ and $A_{j}$.

In Sect. 3 we generalize the definition of $\operatorname{Det}(A)$ to noninvertible operators. Finally, in Sect. 4 we apply our method to the computation of anomalous Jacobians arising from chiral transformations in the path-integral formulation of fermions coupled to $\mathrm{SU}(n)$ gauge fields.

\section{Invertible Operators}

Given an elliptic invertible operator $A$, of order $m>0$ defined on a compact manifold $M$ without boundary, following Seeley [2], we define

$$
A^{s}=\frac{-i}{2 \pi} \int_{\Gamma} \lambda^{s}(\lambda I-A)^{-1} d \lambda,
$$

where $\Gamma$ is a curve beginning at $\infty$, passing along the ray of minimal growth to a small circle about the origin, then clockwise about the circle, and back to $\infty$ along the ray.

We shall denote by $K(x, y, B)$ the kernel of an operator $B$, and $K(x, B)$ this kernel for $x=y$. It can be shown that the generalized $\zeta$-function associated to $A$ (1.1), can be also written as

$$
\zeta(s, A)=\int_{M} \operatorname{Tr} K_{-s}(x, A) d \mu_{x},
$$

where $K_{s}(x, A)=K\left(x, A^{s}\right)$ [2]. (We suppose the volume element $d \mu_{x}$ smooth in each coordinate system.) This alternative definition also applies to non-diagonalizable operators. $\mathscr{C}(M)$ will denote the space of continuous functions on $M$ with norm \|\|$_{\infty}=\sup |f|$.

The main result of this section is the following Theorem:

Theorem. Let $\mathscr{F}$ be a finite-dimensional vector bundle over $M$ ( $M$ a compact manifold without boundary of dimension $n$ ), and let $\Gamma(\mathscr{F})$ be the space of its global sections. Let $A$ be an invertible elliptic pseudo-differential operator of order $m>0$, defined on $\Gamma(\mathscr{F})$ and $A_{1}, \ldots, A_{p}$ differential operators on $\Gamma(\mathscr{F})$ such that $\operatorname{ord}\left(A_{j}\right) \leqq m-1$ for $j=1, \ldots, p$.

If the principal symbol of $A, \sigma_{m}(A)$, satisfies Seeley's hypothesis [2] then

$$
K_{s}\left(x, A+\sum_{i=1}^{p} \varepsilon^{i} A_{i}\right)=K_{s}(x, A)+\sum_{i=1}^{r p} \varepsilon^{i} F_{i}(x, s)+\varepsilon^{r+1} K(x, R(\varepsilon, s)),
$$

where

i) For $-\frac{1}{m}<\operatorname{Re} s<\frac{1}{m}, K_{s}(x, A), F_{i}(x, s)(i=1, \ldots, r p)$ are $\mathscr{C}(M)$-valued analytic functions of $s$.

ii) $F_{1}(x, s)$ is the analytic extension in $s$ of

$$
K\left(x, \frac{i}{2 \pi} \int_{\Gamma} \lambda^{s}(\lambda I-A)^{-1} A_{1}(\lambda I-A)^{-1} d \lambda\right) .
$$


iii) For $\operatorname{Re} s<1 / m,\|K(x, R(\varepsilon, s))\|_{\infty}$ and $\left\|\frac{d}{d s} K(x, R(\varepsilon, s))\right\|_{\infty}$ are bounded for sufficiently small $\varepsilon$.

By integration on $M$ Eq. (2.2) and its derivative with respect to $s$, one gets:

\section{Corollary 1.}

$$
\zeta\left(s, A+\sum_{i=1}^{p} \varepsilon^{i} A_{i}\right)=\zeta(s, A)+\sum_{i=1}^{r p} \varepsilon^{i} f_{i}(s)+\varepsilon^{r+1} f(s, \varepsilon)
$$

where

i) $f_{\hat{i}}(s)$ for $i=1, \ldots, p$, and $f(s, \varepsilon)$ are analytic functions of $s$ if $-\frac{1}{m}<\operatorname{Re}(s)<\frac{1}{m}$.

ii) $f_{1}(s)=\int_{M} \operatorname{Tr}\left(K\left(x, \frac{i}{2 \pi} \int_{\Gamma} \lambda^{-s}(\lambda I-A)^{-1} A_{1}(\lambda I-A)^{-1} d \lambda\right)\right) d \mu_{x}$.

iii) $|f(s, \varepsilon)|$ and $\left|\frac{d}{d s} f(s, \varepsilon)\right|$ are bounded for sufficiently small $\varepsilon$ and $s$ in a compact subset of $\operatorname{Re} s>-\frac{1}{m}$.

\section{Corollary 2.}

i) $\lim _{\varepsilon \rightarrow 0} \zeta^{\prime}\left(s, A+\sum_{i=1}^{p} \varepsilon^{i} A_{i}\right)=\zeta^{\prime}(s, A)$

ii) $\left.\frac{d}{d \varepsilon} \zeta^{\prime}\left(s, A+\sum_{i=1}^{p} \varepsilon^{i} A_{i}\right)\right|_{\varepsilon=0}=f_{1}^{\prime}(s)$.

Proof of the Theorem. We shall denote by $H^{l}, l \in \mathbb{R}$, the Sobolev spaces of global sections of $\mathscr{F}$, and by $\|B\|_{l, j}$ the norm of the operator $B: H^{l} \rightarrow H^{j}$. We set

$$
A_{\varepsilon}^{\prime}=\sum_{i=1}^{p} \varepsilon^{i} A_{i}
$$

For small $\varepsilon$, the operator $A+A_{\varepsilon}^{\prime}$ is also invertible and elliptic, and its principal symbol coincides with $\sigma_{m}(A)$. Then

$$
\left(A+A_{\varepsilon}^{\prime}\right)^{s}=\frac{-i}{2 \pi} \int_{\Gamma} \lambda^{s}\left(\lambda I-\left(A+A_{\varepsilon}^{\prime}\right)\right)^{-1} d \lambda, \quad \operatorname{Re} s<0 .
$$

We shall expand this operator in powers of $\varepsilon$. Then we write

$$
\begin{aligned}
{\left[\lambda I-\left(A+A_{\varepsilon}^{\prime}\right)\right]^{-1} } & =\left[I-(\lambda I-A)^{-1} A_{\varepsilon}^{\prime}\right]^{-1}(\lambda I-A)^{-1} \\
& =\sum_{n=0}^{\infty}\left[(\lambda I-A)^{-1} A_{\varepsilon}^{\prime}\right]^{n}(\lambda I-A)^{-1}
\end{aligned}
$$

This expansion holds for $\lambda \in \Gamma$ and $|\varepsilon| \leqq \varepsilon_{0}$, with sufficiently small $\varepsilon_{0}$ such that $\left\|(\lambda I-A)^{-1} A_{\varepsilon}^{\prime}\right\|_{l, l}<1$. Note that $\varepsilon_{0}$ can be chosen independently of $\lambda$, for $\lambda$ on $\Gamma$, 
because

$$
\begin{aligned}
\left\|(\lambda I-A)^{-1} A_{\varepsilon}^{\prime}\right\|_{l, l} & \leqq \sum_{k=1}^{p} \varepsilon^{k}\left\|(\lambda I-A)^{-1} A_{k}\right\|_{l, l} \\
& \leqq \sum_{k=1}^{p} \varepsilon^{k}\left\|(\lambda I-A)^{-1}\right\|_{l-(m-1), l}\left\|A_{k}\right\|_{l, l-(m-1)} .
\end{aligned}
$$

Now, for $0 \leqq p \leqq m$, there exists (see [2]) a positive constant $C_{p}$ such that

$$
\left\|(\lambda I-A)^{-1}\right\|_{j, j+p} \leqq C_{p}|\lambda|^{-1+p / m},
$$

if $\lambda$ is large enough, say $|\lambda|>C$ for suitable positive constant $C$. Hence, taking $p=m-1$

$$
\left\|(\lambda I-A)^{-1} A_{j}\right\|_{l, l} \leqq C_{l}|\lambda|^{-1 / m} \text { for } \quad|\lambda|>C .
$$

On the other hand, $\left\|(\lambda I-A)^{-1}\right\|_{j, j+p}$ is continuous in $\lambda$ for $\lambda \notin \operatorname{Sp}(A)$, then it is uniformly bounded for $\lambda$ on $\Gamma$ and $|\lambda| \leqq C$.

Now, we write

$$
\begin{aligned}
{\left[\lambda I-\left(A+A_{\varepsilon}^{\prime}\right)\right]^{-1}=} & (\lambda I-A)^{-1}+\sum_{n=1}^{r}\left[(\lambda I-A)^{-1} A_{\varepsilon}^{\prime}\right]^{n}(\lambda I-A)^{-1} \\
& +\left[(\lambda I-A)^{-1} A_{\varepsilon}^{\prime}\right]^{r+1}\left(\lambda I-\left(A+A_{\varepsilon}^{\prime}\right)\right)^{-1}
\end{aligned}
$$

where

$$
\left[(\lambda I-A)^{-1} A_{\varepsilon}^{\prime}\right]^{n}=\left[\sum_{j=1}^{p} \varepsilon^{j}(\lambda I-A)^{-1} A_{j}\right]^{n}=\sum_{j=n}^{n p} \varepsilon^{j} B_{j}^{n}(\lambda) .
$$

The operators $B_{j}^{n}(\lambda)$ are sums of finite products of the $(\lambda I-A)^{-1} A_{j}$ and therefore bounded operators from $H^{l}$ to $H^{l}$.

Hence

$$
\begin{aligned}
\left(A+A_{\varepsilon}^{\prime}\right)^{s}= & \frac{-i}{2 \pi} \int_{\Gamma} \lambda^{s}(\lambda I-A)^{-1} d \lambda+\sum_{n=1}^{r} \frac{-i}{2 \pi} \int_{\Gamma} \lambda^{s}\left[(\lambda I-A)^{-1} A_{\varepsilon}^{\prime}\right]^{n}(\lambda I-A)^{-1} d \lambda \\
& +\frac{-i}{2 \pi} \int_{\Gamma} \lambda^{s}\left((\lambda I-A)^{-1} A_{\varepsilon}^{\prime}\right)^{r+1}\left(\lambda I-\left(A+A_{\varepsilon}^{\prime}\right)\right)^{-1} d \lambda \\
= & A^{s}+\sum_{j=1}^{r p} \varepsilon^{j} \frac{i}{2 \pi} \int_{\Gamma} \lambda^{s} C_{j}(\lambda)(\lambda I-A)^{-1} d \lambda \\
& +\varepsilon^{r+1} \frac{i}{2 \pi} \int_{\Gamma} \lambda^{s}\left[(\lambda I-A)^{-1}\left(\sum_{j=1}^{p} \varepsilon^{j-1} A_{j}\right)\right]^{p+1}\left[\lambda I-\left(A+A_{\varepsilon}^{\prime}\right)\right]^{-1} d \lambda
\end{aligned}
$$

where each $C_{j}(\lambda)$ is a sum of operators $B_{j}^{n}(\lambda)$.

We have that

$$
T_{j}(s)=\frac{-i}{2 \pi} \int_{\Gamma} \lambda^{s} C_{j}(\lambda)(\lambda I-A)^{-1} d \lambda
$$

is a well defined operator for $\operatorname{Re} s<0$, because

$$
\left\|C_{j}(\lambda)(\lambda I-A)^{-1}\right\|_{l, l}<\left\|C_{j}(\lambda)\right\|_{l, l}\left\|(\lambda I-A)^{-1}\right\|_{l, l}<C|\lambda|^{-1}
$$


for sufficiently large $\lambda$. By (2.4) for $p=0,\left\|C_{j}(\lambda)\right\|_{l, i}$ is uniformly bounded if $\lambda$ on $\Gamma$ since so is $\left\|(\lambda I-A)^{-1} A_{i}\right\|_{l, l}$. Also $T_{j}(s)$ are Calderón-Zygmund operators (as they are considered by Seeley in [2]). Thus, if $\operatorname{Re} s<\frac{1-n}{m}, T_{j}(s)$ is an integral operator whose kernel $K\left(x, y, T_{j}(s)\right)$ is continuous for $x=y$, since $\operatorname{Re}\left(\right.$ ord $\left.T_{j}(s)\right)<\operatorname{Re}(m s-1)$.

In the last term of $(2.5)$

$$
R(s, \varepsilon)=\frac{-i}{2 \pi} \int_{\Gamma} \lambda^{s}\left[(\lambda I-A)^{-1}\left(\sum_{j=1}^{p} \varepsilon^{j-1} A_{j}\right)\right]^{r+1}\left(\lambda I-\left(A+A_{\varepsilon}^{\prime}\right)\right)^{-1} d \lambda .
$$

By (2.4) taking $p=m, p=m-1$, and $p=0$, we see that

$$
\begin{gathered}
\left\|\left[(\lambda I-A)^{-1}\left(\sum_{j=1}^{p} \varepsilon^{j-1} A_{j}\right)\right]^{r}\right\|_{l, l+r}<C_{l}, \\
\left\|(\lambda I-A)^{-1}\left(\sum_{j=1}^{p} \varepsilon^{j-1} A_{j}\right)\right\|_{l, l}<C_{l}|\lambda|^{-1 / m},
\end{gathered}
$$

and

$$
\|\left(\lambda I-\left(A+A_{\varepsilon}^{\prime}\right)^{-1}\left\|_{l, l}=\right\|\left(I-(\lambda I-A)^{-1} A_{\varepsilon}^{\prime}\right)^{-1}(\lambda I-A)^{-1} \|_{l, l}<C_{l}|\lambda|^{-1},\right.
$$

for sufficiently small $\varepsilon$ (the constants $C_{l}$ depend only on $l, r, A, A_{j}$ and not on $\varepsilon$ ).

From the above estimates we deduce that the norm \|\|$_{l, l+r}$ of the integrand in (2.6) is bounded by $C|\lambda|^{-1-1 / m}$. Hence $R(s, \varepsilon)$ is a bounded operator from $H^{i}$ to $H^{i+r}$ and it is well defined for $\operatorname{Re} s<\frac{1}{m}$. If one chooses $r>n, R(s, \varepsilon)$ has continuous kernel because $\delta_{x} \in H^{-\{n / 2)-\alpha}(\alpha>0)$ (where $\delta_{x}$ is the Dirac $\delta$-function at the point $x$ in $M$ ), so $R(s, \varepsilon) \delta_{y} \in H^{-(n / 2)-\alpha+r} C H^{(n / 2)+\alpha}$, and then $K(x, y, R(s, \varepsilon))=\left\langle\delta_{x}, R(s, \varepsilon) \delta_{y}\right\rangle$ verifies: 1) is a well defined continuous function of $x, y ; 2)$ is a $\mathscr{C}(M \times M)$ valued analytic function of $s ; 3$ ) it is bounded function for small $\varepsilon$ since $\|R(s, \varepsilon)\|_{-(n / 2)-\alpha,(n / 2)+\alpha}<C$ for such $\left.\varepsilon ; 4\right) \frac{d}{d s} K(x, R(s, \varepsilon))=K\left(x, \frac{d}{d s} R(s, \varepsilon)\right)$ since $R(s, \varepsilon)$ is an analytic function of $s$ for $\operatorname{Re} s<\frac{1}{m}$ valued in the space of continuous operator from $H^{l}$ to $H^{l+v}$ and ; 5) $\left\|\frac{d}{d s} R(s, \varepsilon)\right\|_{-(n / 2)-\alpha_{(}(n / 2)+\alpha}$ is uniformly bounded for small $\varepsilon$.

We have then the estimates iii) of the theorem.

From the above discussion and (2.5) we have

$$
K_{s}\left(x, A+A_{\varepsilon}^{\prime}\right)=K_{s}(x, A)+\sum_{j=1}^{r p} \varepsilon^{j} K\left(x, T_{j}(s)\right)+\varepsilon^{r+1} K(x, R(s, \varepsilon)) .
$$

This equation has been proved for $\operatorname{Re} s<-\frac{n}{m}$, since $\left(A+A_{\varepsilon}^{\prime}\right)^{s}, A^{s}$ and $T_{j}(s)$ have continuous kernels for these values of $s$. Moreover, $K_{s}\left(x, A+A_{\varepsilon}^{\prime}\right)$ and $K_{s}(x, A)$ 
admit meromorphic extensions to the whole complex plane with poles only at $s=\frac{-n+j}{m}, j \in \mathbb{N}$, unless they are non-negative integers, and $K(x, R(s, \varepsilon))$ is analytic in $s$ for $\operatorname{Re} s<\frac{1}{m}$. These continuations will be denoted by $F_{j}(x, s)$.

Finally, it is easy to see that

$$
T_{1}(s)=\frac{i}{2 \pi} \int_{\Gamma} \lambda^{s}(\lambda I-A)^{-1} A_{1}(\lambda I-A)^{-1} d \lambda .
$$

Thus ii) follows. Q.E.D.

Remark. We don't know if the hypothesis ord $A_{j}<m$ can be dropped.

Summarizing, our main result is

$$
\operatorname{Det}\left(A+A_{\varepsilon}^{\prime}\right)=\operatorname{Det}(A) \exp -\left[\varepsilon f_{1}^{\prime}(0)+\varepsilon^{2} f_{2}^{\prime}(0)+\ldots+\varepsilon^{r} f_{r}^{\prime}(0)+0\left(\varepsilon^{r+1}\right)\right],
$$

where

$$
f_{j}(s)=\int_{M} \operatorname{Tr}\left(F_{j}(x,-s)\right) d \mu_{x},
$$

with

$$
F_{j}(x, s)=K\left(x, T_{j}(s)\right)=K\left(x, \frac{i}{2 \pi} \int_{\Gamma} \lambda^{s} C_{j}(\lambda)(\lambda I-A)^{-1} d \lambda\right)
$$

$C_{j}(\lambda)=\sum_{n=1}^{j} B_{j}^{n}(\lambda)$, and $B_{j}^{n}(\lambda)$ the coefficient of $\varepsilon^{j}$ in the expansion of

$$
\left[\varepsilon(\lambda I-A)^{-1} A_{1}+\varepsilon^{2}(\lambda I-A)^{-1} A_{2}+\ldots+\varepsilon^{p}(\lambda I-A)^{-1} A_{p}\right]^{n} .
$$

Remark. If $\operatorname{Res}$ is large enough to have $\beta_{s}=\operatorname{Re}[m(-s-1)]+\operatorname{ord} A_{1}<-n$, $A^{-s-1} A_{1}$ is an integral operator with continuous kernel, and then of trace class, since it is pseudo-differential of order $\beta_{s^{\prime}}$. So, for these values of $s$

$$
f_{1}(s)=-s \operatorname{Tr}\left(A^{-s-1} A_{1}\right)
$$

[if $s$ is such that $\beta_{s}>-n, f_{1}(\mathrm{~s})$ is the analytic continuation of the right hand term in (2.8)].

Analogously, if $A_{1}, \ldots, A_{j}$ commute with $A$, the function $f_{j}(s)$ can also be considered as a trace. For instance, if $A_{1}, A_{2}$ commute with $A$,

$$
\begin{aligned}
f_{2}(s)= & \operatorname{Tr}\left[\frac { i } { 2 \pi } \int _ { \Gamma } \lambda ^ { - s } \left((\lambda I-A)^{-1} A_{2}(\lambda I-A)^{-1}\right.\right. \\
& \left.\left.+(\lambda I-A)^{-1} A_{1}(\lambda I-A)^{-1} A_{1}(\lambda I-A)^{-1}\right) d \lambda\right] \\
= & \operatorname{Tr}\left[-s A^{-s-1} A_{2}\right]+\operatorname{Tr}\left[-s(-s-1) A^{-s-2} A_{1}^{2}\right],
\end{aligned}
$$

if

$$
\operatorname{Re}[m(-s-1)]+\operatorname{ord} A_{2}<-n,
$$


and

$$
\operatorname{Re}[m(-s-2)]+2 \operatorname{ord} A_{1}<-n \text {. }
$$

Example. For $M=S^{1}, A=-\frac{d^{2}}{d x^{2}}+P$, being $P$ the orthogonal projection on the constants, $p=1$ and $A_{1}=i \frac{d}{d x}$. We have that $\zeta(s, A)=1+2 \zeta(2 s)[\zeta(2 s)$ is the numerical Riemann $\zeta$-function], and then $\operatorname{Det}(A)=(2 \pi)^{2}$.

We obtain

$$
\log \operatorname{Det}\left(A+\varepsilon A_{1}\right)=\log \operatorname{Det}(A)-\sum_{k=1}^{r} \frac{\varepsilon^{2 k}}{k} \zeta(2 k)+R_{r},
$$

since

$$
f_{k}^{\prime}(0)= \begin{cases}0 & \text { if } k \text { is odd } \\ \frac{2}{k} \zeta(k) & \text { if } k \text { is even. }\end{cases}
$$

It can be proved that $R_{\substack{r \rightarrow 0 \\ r \rightarrow \infty}}$, and then

$$
\operatorname{Det}\left(A+\varepsilon A_{1}\right)=\frac{(2 \pi)^{2}}{\Gamma(1-\varepsilon) \Gamma(1+\varepsilon)} .
$$

\section{Non-Invertible Operators}

The above definitions and proofs hold only for an invertible operator $A$. Otherwise, i.e. when $A$ admits $\lambda=0$ as eigenvalue, any natural definition for determinant of $A$ should vanish. However, it is interesting to keep the "product" of the other eigenvalues. This fact leads us to introduce the following generalized definition

$$
\operatorname{Det}^{\prime}(A)=\lim _{\alpha \rightarrow 0} \frac{\operatorname{Det}(A+\alpha I)}{\alpha^{N}},
$$

where $\alpha$ is a real positive parameter, and $N=\operatorname{dim} \operatorname{Ker} A$ (i.e. the dimension of the null set of $A$ ). We are going to prove that (3.1) is an adequate definition: we shall see that $\operatorname{Det}^{\prime}(A)$ coincides with $\operatorname{Det}(A)$ if $A$ is invertible, and in general, it coincides with $\operatorname{Det}\left(A+P_{\mathrm{ker} A}\right)$, where $P_{\mathrm{ker} A}$ is the orthogonal projection on $\operatorname{Ker} A$. $\operatorname{Det}^{\prime}(A)$ can also be thought of as the regularized determinant of the operator $A /(\operatorname{Ker} A)^{\perp}$.

Proposition. Let $A$ be as in the theorem in the previous section, but not necessary invertible; if $A$ admits a complete orthogonal set of eigenfunctions, then the limit (3.1) exists and

$$
\operatorname{Det}^{\prime}(A)=\operatorname{Det}\left(A+P_{\mathrm{ker} A}\right) \text {. }
$$

Proof. If $N=0$, the result follows from Corollary 2 , taking $p=1$ and $A_{1}=I$. 
If $N>0$, note that for sufficiently small $\alpha, A+\alpha I$ is invertible and then $\operatorname{Det}(A+\alpha I)$ is well defined. On the other hand, we know that for $\operatorname{Res}>\frac{n}{m}$

$$
\zeta(s, A+\alpha I)=\sum_{\lambda_{j} \neq 0}\left(\lambda_{j}+\alpha\right)^{-s}+N \alpha^{-s},
$$

and

$$
\zeta\left(s, A+P_{\mathrm{ker} A}+\alpha I\right)=\sum_{\lambda_{j} \neq 0}\left(\lambda_{j}+\alpha\right)^{-s}+N(1+\alpha)^{-s},
$$

then

$$
\zeta(s, A+\alpha I)-N \alpha^{-s}=\zeta\left(s, A+P_{\mathrm{ker} A}+\alpha I\right)-N(1+\alpha)^{-s} .
$$

Since both terms have analytic continuations to a neighbourhood of $s=0$, and from the continuity established in Corollary 2-i) we have

$$
\lim _{\alpha \rightarrow 0} \zeta^{\prime}(0, A+\alpha I)+N \ln \alpha=\lim _{\alpha \rightarrow 0} \zeta^{\prime}\left(0, A+P_{\mathrm{ker} A}+\alpha I\right)=\zeta^{\prime}\left(0, A+P_{\mathrm{ker} A}\right) . \quad \text { Q.E.D. }
$$

Note that for $N=0$ the hypothesis on the existence of a complete orthonormal set of eigenfunctions is unnecessary.

\section{An Application of the Method: Chiral Changes in Fermionic Variables}

Recently Fujikawa [3] has shown that the Euclidean path-integral measure for gauge theories with fermions is not invariant under chiral transformations, and that it gives rise to a Jacobian related to the Adler-Bell-Jackiw anomaly [4]. In Fujikawa's work the Jacobian arising from the $\gamma_{5}$-transformation was regularized by means of some particular procedure, but such a choice appears not to be completely justified. More recently Andrianov, Bonora and one of us [5] have stressed the role played by the gauge invariance in Fujikawa's choice of the regularization procedure.

In this section we are going to apply the approach we developed in the preceding sections in order to give a more rigorous derivation of Fujikawa's result and to clarify some related points.

We are going to assume that the fields behave at the infinite so that it is possible to compactify to $S^{n}$ by stereographic projection (see for example [6]).

Let us consider the "partition function" for Dirac fields coupled to an external $\mathrm{SU}(N)$ gauge field $A_{\mu}$

$$
Z(\not D)=\int \mathscr{D} \bar{\Psi} \mathscr{D} \Psi \exp \left\{-\int \bar{\Psi}(\not D-i m) \Psi d x\right\}
$$

where the Euclidean Dirac operator is $\not D=-i \gamma_{\mu}\left(\partial^{\mu}+A^{\mu}\right)=\not D^{*}$, with $A_{\mu}=T^{a} A_{\mu}^{a}$ and $\gamma_{\mu}^{*}=\gamma_{\mu},\left(T^{a}\right.$ are the $N^{2}-1$ antihermitian generators of the gauge group). We shall denote by $D_{m}$ the invertible operator $D_{m}=\not D-i m$.

According to Berezin's integration rules [7], the path-integral in Eq. (4.1) is formally the determinant of the operator $D_{m}$. But, as it is well known, it diverges. 
Then, it must be regularized preserving the gauge invariance of the theory. Under a gauge transformation $\Omega$, we have

$$
\begin{gathered}
A_{\mu} \rightarrow \Omega A_{\mu} \Omega^{-1}-\left(\partial_{\mu} \Omega\right) \Omega^{-1}, \\
\not D \rightarrow \Omega \not D \Omega^{-1}, \\
\Psi \rightarrow \Omega \Psi \text { and } \bar{\Psi} \rightarrow \bar{\Psi} \Omega^{-1} .
\end{gathered}
$$

Then we have to demand that

$$
Z_{\mathrm{r} c \mathrm{~g}}\left(\Omega \not D \Omega^{-1}\right)=Z_{\mathrm{reg}}(\not D) .
$$

We can adopt the $\zeta$ function regularization technique [1]:

$$
Z_{\mathrm{reg}}\left(D_{m}\right)=\left[\operatorname{Det}\left(D_{m} D_{m}^{*}\right)\right]^{1 / 2}=\exp \left(-\frac{1}{2} \zeta^{\prime}\left(0, \not D^{2}+m^{2} I\right)\right)
$$

since if we take into account that the eigenvalues of $\Omega D_{m} \Omega^{-1}$ and $D_{m}$ coincide, it follows immediately from the series (1.1) that

$$
\operatorname{Det}\left(\Omega D_{m} D_{m}^{*} \Omega^{-1}\right)=\operatorname{Det}\left(D_{m} D_{m}^{*}\right) .
$$

If we perform the infinitesimal linear change of variables:

$$
\Psi=\Omega_{5} \chi \quad \text { and } \quad \bar{\Psi}=\bar{\chi} \Omega_{5},
$$

where $\Omega_{5}=1+\varepsilon \gamma_{5} \phi(x)$, in the path integral defining $Z\left(D_{m}\right)$ of Eq. (4.1), we shall obtain

$$
\operatorname{Det}\left(D_{m} D_{m}^{*}\right)=J^{2} \operatorname{Det}\left(\Omega_{5} D_{m} \Omega_{5}^{2} D_{m}^{*} \Omega_{5}\right),
$$

where $J$ is the "Jacobian" associated to the linear transformation (4.3). If the operators were finite-dimensional, the usual properties of determinants would lead to

$$
\log J=-2 \log \operatorname{det}\left(\Omega_{5}\right)=-2 \varepsilon \operatorname{Tr}\left(\gamma_{5} \phi\right),
$$

and the Jacobian would depend on nothing but $\Omega_{5}$. This will not necessarily occur in the $\infty$-dimensional case: through the regularization the Jacobian can depend on $\not D$ too. That is the reason we call them anomalous Jacobians. Note that in our approach $J$ is finite from the beginning, since it is the quotient of two regularized determinants, as opposed to Fujikawa's method where it must be regularized.

Under the transformation (4.3), the operator $D_{m}$ transforms as

$$
D_{m} \rightarrow \Omega_{5} D_{m} \Omega_{5}=D_{m}+\varepsilon\left(\gamma_{5} \phi D_{m}+D_{m} \phi \gamma_{5}\right)+O\left(\varepsilon^{2}\right) .
$$

Then

$$
\begin{aligned}
\Omega_{5} D_{m} \Omega_{5}^{2} D_{m}^{*} \Omega_{5}= & D_{m} D_{m}^{*}+\varepsilon\left(2 D_{m} \gamma_{5} \phi D_{m}^{*}+D_{m} D_{m}^{*} \gamma_{5} \phi+\gamma_{5} \phi D_{m} D_{m}^{*}\right) \\
& +O\left(\varepsilon^{2}\right)=\not D^{2}+m^{2} I+\varepsilon A_{1}+O\left(\varepsilon^{2}\right)
\end{aligned}
$$

Therefore, from (4.2), (4.4), and (4.6), we obtain

$$
\log J=\frac{1}{2} \zeta^{\prime}\left(0,\left(\not D^{2}+m^{2} I\right)+\varepsilon A_{1}\right)-\frac{1}{2} \zeta^{\prime}\left(0, \not D^{2}+m^{2} I\right) .
$$


Using now the results of the corollaries of Sect. 2, we obtain

$$
\begin{aligned}
\log J & =-\frac{\varepsilon}{2} \frac{d}{d s}\left[s \operatorname{Tr}\left\{\left(\not D^{2}+m^{2} I\right)^{-s-1}\left(2 D_{m} \gamma_{5} \phi D_{m}^{*}+D_{m} D_{m}^{*} \gamma_{5} \phi+\gamma_{5} \phi D_{m} D_{m}^{*}\right)\right\}\right]_{s=0} \\
& =-2 \varepsilon \frac{d}{d s}\left[s \operatorname{Tr}\left\{\left(\not D^{2}+m^{2} I\right)^{-s} \gamma_{5} \phi\right\}\right]_{s=0} .
\end{aligned}
$$

$\operatorname{Tr}\{\ldots\}$ can be written as

$$
\int_{M} \operatorname{tr}\left(K_{-s}\left(x, \not D^{2}+m^{2} I\right) \gamma_{5} \phi\right) d \mu_{x}
$$

where tr stands for the trace of the color and $\gamma$-matrices. Since $K_{-s}$ is analytic in a neighbourhood of $s=0, s \frac{d}{d s} \operatorname{Tr}\{\ldots\} \underset{s \rightarrow 0}{\longrightarrow} 0$. Then, (4.7) reads

$$
\log J=-\left.2 \varepsilon \operatorname{Tr}\left\{\left(\not D^{2}+m^{2} I\right)^{-s} \gamma_{5} \phi\right\}\right|_{s=0} .
$$

Now, it is clear what is going on. The naive limit would lead to Eq. (4.5) as in the finite-dimensional case. But the trace of $\gamma_{5} \phi(x)$ does not exist, so (4.8) must be evaluated for sufficiently large $\operatorname{Re} s$, and then analytically extended to $s=0$. Roughly speaking, one can say that the operator $\left(\not D^{2}+m^{2} I\right)^{-s}$ regularizes the trace. In this process some dependence on this operator could, and it will, remain after the limit. It is important to notice that, in our evaluation of $J$, the regularizing operator has appeared naturally by adopting from the beginning the gauge invariant $\zeta$-function regularization procedure.

If we write

$$
\not D=\left(\begin{array}{cc}
0 & D^{-} \\
D^{+} & 0
\end{array}\right)
$$

(4.8) gives

$$
\log J=-2 \varepsilon \int_{M}\left[K_{0}\left(D^{-} D^{+}+m^{2}\right)-K_{0}\left(D^{+} D^{-}+m^{2}\right)\right] \phi(x) d \mu_{x} .
$$

The $K_{0}$ 's can be evaluated after a rather lengthy but straightforward computation by means of Seeley's [2] formulae, taking into account that

$$
\not D^{2}=-D_{\mu} D_{\mu}+\frac{1}{4}\left[\gamma^{\mu}, \gamma^{\nu}\right] F_{\mu \nu} .
$$

Then, we finally reobtain Fujikawa's result for $\mathrm{QCD}_{4}$ :

$$
\log J=-\frac{\varepsilon}{8 \pi^{2}} \int \operatorname{tr} F_{\mu v}^{*} F^{\mu v} \phi d x .
$$

In the case of $\mathrm{QED}_{2}$ the anomalous Jacobian satisfies

$$
\log J=-\frac{\varepsilon}{2 \pi} \int \varepsilon_{\mu v} F_{\mu \nu} \phi d x .
$$

Note that (4.10) and (4.11) could also have been obtained from (4.8) as an application of Atiyah, Bott, and Patodi results [8]. 
Acknowledgements. We are very grateful to Proffessor A.P. Calderón and D. Alvarez Alonso for helpful discussions and critical remarks. One of the authors (R.E.G.S.) is indebted to A. Andrianov, L. Bonora, R. Jackiw, and F. Schaposnik for helpful conversations about Fujikawa's derivation of anomalies.

\section{References}

1. Hawking, S.W.: Zeta function regularization of path integrals in curved spacetime. Commun. Math. Phys. 55, 133-148 (1977)

2. Seeley, R.T.: Am. Math. Soc. Proc. Symp. Pure Math. 10, 288-307 (1967)

3. Fuikawa, K.: Path-integral measure for gauge-invariant fermion fields. Phys. Rev. Lett. 42. 1195 (1979); Path integral for gauge theories with fermions. Phys. Rev. D21, 2848 (1980)

4. Adler, S: Axial-vector vertex in spinor electrodynamics. Phys. Rev. 177, 2426 (1969) Bell, J, Jackiw, R. A PCAC puzzle: $\pi^{\circ} \rightarrow y$ in the $\sigma$-model. Nuovo Cimento 60 A, 47 (1969)

5. Andrianov, A., Bonora, L, Gamboa-Saravi, R.: Phys. Rev. D26, 2821 (1982)

6. Nash, C.: The theory and practice of zeta functions. TCD-1979-2

7. Berezin, F.: The method of second quantization. New York: Academic Press 1969

8. Atiyah, M., Bott, R., Patodi, V.K. : Invent. Math. 19, 279-330 (1973)

9. Muschietti, M.A.: Tesis, Universidad Nacional de La Plata (1983)

Communicated by H. Araki

Received September 25, 1982

Note added in proof: After we sent this paper for publication, one of us (M.A.M.) has proved that if $\left\{A_{\varepsilon}\right\}$ is a family of pseudodifferential operators depending analytically on the parameter $\varepsilon$ and ord $A_{c} \leqq$ ord $A_{0}=m$ for small $\varepsilon$, then $K_{s}\left(x, x, A_{\varepsilon}\right)$ is an analytic function of $\varepsilon$, in a neighbourhood of $\varepsilon=0$, for $\operatorname{Res}<\frac{1}{m}$. Nevertheless, in this case, a formula such as $(2.8)$ has not been found [9]. 